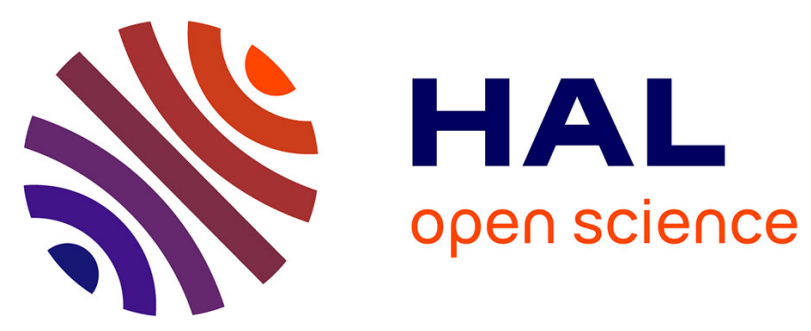

\title{
Measurement of Three-dimensional Mirror Parameters by Polarization Imaging applied to Catadioptric Camera Calibration
}

Olivier Morel, Ralph Seulin, David Fofi

\section{- To cite this version:}

Olivier Morel, Ralph Seulin, David Fofi. Measurement of Three-dimensional Mirror Parameters by Polarization Imaging applied to Catadioptric Camera Calibration. Journal of Electronic Imaging, 2008, 17 (3), pp.031105. 10.1117/1.2958290 . hal-00608492

\section{HAL Id: hal-00608492 \\ https://hal.science/hal-00608492}

Submitted on 13 Jul 2011

HAL is a multi-disciplinary open access archive for the deposit and dissemination of scientific research documents, whether they are published or not. The documents may come from teaching and research institutions in France or abroad, or from public or private research centers.
L'archive ouverte pluridisciplinaire HAL, est destinée au dépôt et à la diffusion de documents scientifiques de niveau recherche, publiés ou non, émanant des établissements d'enseignement et de recherche français ou étrangers, des laboratoires publics ou privés. 


\title{
Measurement of three-dimensional mirror parameters by polarization imaging applied to catadioptric camera calibration
}

\author{
Olivier Morel, Ralph Seulin, David Fofi
}

\begin{abstract}
A new efficient method of calibration for catadioptric sensors is presented in this paper. The method is based on an accurate measurement the three-dimensional parameters of the mirror by means of polarization imaging. While inserting a rotating polarizer between the camera and the mirror, the system is automatically calibrated without any calibration patterns. Moreover, this method permits most of the constraints related to the calibration of catadioptric systems to be relaxed. We show that, contrary to our system, the traditional methods of calibration are very sensitive to misalignment of the camera axis and the symmetry axis of the mirror. From the measurement of three-dimensional parameters, we apply the generic calibration concept to calibrate the catadioptric sensor. The influence of the disturbed measurement of the parameters on the reconstruction of a synthetic scene is also presented. Finally, experiments prove the validity of the method with some preliminary results on three-dimensional reconstruction.
\end{abstract}

PACS numbers: 


\section{INTRODUCTION}

Conventional perspective cameras have limited fields of view that make them restrictive in some applications such as robotics, video-surveillance, and so on. One way to enhance the field of view is to place a mirror with a surface of revolution in front of the camera so that the scene reflects on the mirror omnidirectionally. Such a system, comprised of both lenses (dioptric) and mirrors (catoptric) for image formation, is called catadioptric. Several configurations exist and those satisfying the single viewpoint constraint are described in [1].

Catadioptric vision systems available on the market have been extensively studied. Commercial devices are not adapted to our requirements, because optical components need to be placed between the camera and the mirror. Mirrors have therefore been produced in our own facilities thanks to the Plateform3D department [32]. A high speed machining centre has been used to produce a very high quality surface, which is polished after production.

We developed a new approach of calibrating catadioptric sensors by polarization imaging. This method enables the calibration of any mirror shape, since it is based on the measurement of three-dimensional parameters such as height and normal orientations of the surface. The only constraint is that an orthographic camera has to be used. To calibrate the system, we apply the generic calibration concept developed by Sturm and Ramalingam $[2,3]$.

The article is structured as follows. The next section recalls previous work on paracatadioptric calibration, since the measurement of the surface normals by polarization imaging induces orthographic projection, and most of the calibration methods developed for catadioptric systems rely on the single viewpoint constraint. We show the misalignment sensitivity of these methods for the reconstruction of a synthetic scene. Then, after presenting some basic knowledge about polarization imaging, we detail how to calibrate the sensor with the generic calibration concept. In section 4, simulations are presented to illustrate the influence of the parameter measurement on the quality of the reconstruction. Preliminary results on a calibrated spherical mirror are also described. The paper ends with a conclusion and a few words about future work to be undertaken. 


\section{CALIBRATION OF CATADIOPTRIC CAMERAS}

\section{A. Previous work}

The most obvious calibration method that can be used is an approach based on the image of the mirror's bounding circle $[4,5]$. It has the main advantage of being easily automated, but the drawbacks are that the surface mirror has to be very accurate and the mirror boundary has to accurately encode the intrinsic parameters. Other self-calibration method can be found in $[6,7]$. Another approach of calibrating catadioptric sensor is to use geometric invariants on the image such as lines or circles $[8,9,10,11]$. Finally the sensor can also been calibrated by using some calibration pattern with control points whose 3D world coordinates are known $[12,13,14,15,16]$.

Since our approach requires a telecentric lens, we recall some methods devoted to paracatadioptric camera calibration (the Single View Point constraint involve a telecentric lens and a paraboloidal mirror). In this case, the more robust methods are based on the fitting of lines projected onto the mirror $[8,17,18]$. This approach also has some shortcomings: lines have to be precisely detected and the optical axis of the camera is assumed to be aligned with the symmetry axis of the paraboloid. To illustrate the misalignment effect, the three-dimensional reconstructions of a synthetic scene based on calibrations using the three preceding methods are simulated (Figure 1).

The scene represents a room of size $500 \times 500 \times 250 \mathrm{~cm}$ with elements such as windows, doors, and a table. Three images of the catadioptric sensor are used to triangulate the points of the scene. For the calibration process, 20 lines are computed and perfectly detected on the mirror. Then the calibration parameters are used to reconstruct the scene, according to the linear-eigen method [19]. As presented in Figure 2, the misalignment of the paracatadioptric system leads to the introduction of an important error on the reconstruction even if the calibration is performed with perfect line fittings.

To deal with the alignment errors between the mirror and the lens, more sophisticated algorithms were introduced $[20,21,22]$. In [20, 21], the calibration process, which requires a large number of parameters to estimate, involves a three step algorithm in order to compute all the extrinsic and intrinsic parameters. In [22], a more flexible solution based on caustic curves is proposed: the main advantage of this method is that it is applicable for every surface 


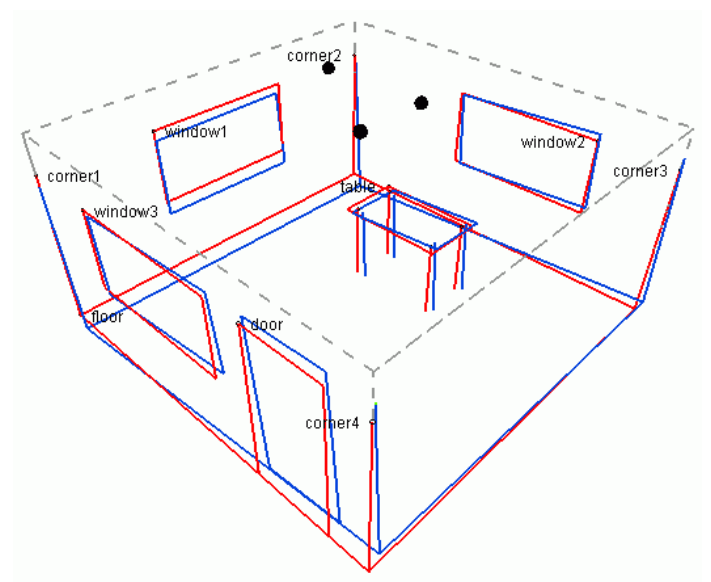

Figure 1: Simulation of the three-dimensional reconstruction: the theoretical scene, in blue, represents a room with elements such as windows, door and table ; black dots depict the three locations of the sensor. The reconstructed scene with the Vanderportaele calibration method (misalignment of $2^{\circ}$ ) is drawn in red.

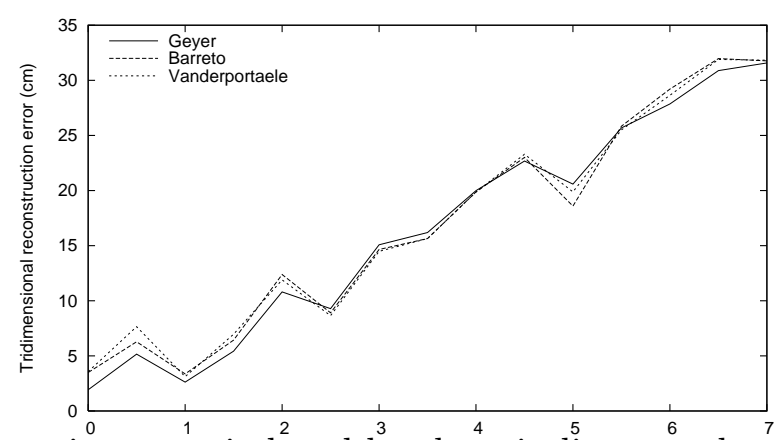

Figure 2: Reconstruction error induced Nayngtatemisalignment between the optical axis of the camera and the symmetry axis of the paraboloid. Calibration method used are Geyer, Barreto and Vanderportaele. (room of size $500 \times 500 \times 250 \mathrm{~cm}$ )

of revolution. In order to relax constraints on the mirror shape, the method presented in this paper is based on the generic calibration concept: it leads to a flexible, easy-to-perform, and shape-independent method of calibration.

\section{B. The generic calibration concept}

The previous calibration methods for omnidirectional catadioptric sensors assume that: (i) the mirror shape is perfectly known; (ii) the alignment of the sensor is perfect so that 
the single viewpoint constraint is satisfied; and (iii) the projection model can be easily parameterized. Some methods relax the second constraint and a few relax the first one, but before recent work $[3,12,23]$ calibrating methods always underlie an explicit parametric model of projection. This new model has the advantage of working for any type of camera (catadioptric systems, central cameras with or without distortion, axial cameras, etc.) and of handling heterogeneous systems [2] (for instance, a sensor composed of an omnidirectional camera and a perspective camera). However, developing an efficient and easy-to-use calibration method based on this model is not trivial. In this paper, the proposed new method enables catadioptric sensor calibration by polarization imaging. It relaxes the three constraints listed above ((i), (ii), and (iii)). Moreover, the calibration can even be performed by a non-specialist as it only requires an optical apparatus and no image processing.

\section{POLARIZATION IMAGING}

Polarization imaging enables the study of the polarization state of a light wave. The most common applications in artificial vision involve segmenting dielectric and metallic objects [24] and to detect transparent surfaces. Polarization imaging likewise enables three-dimensional information of specular objects to be detected ("Shape from polarization" method [25, 26]). The physical principle behind "Shape from polarization" is the following: after being reflected, an unpolarized light wave becomes partially linearly polarized, depending on the surface normal and the refractive index of the media it impinges on. Partially linearly polarized light has three parameters: the light magnitude $I$, the degree of polarization $\rho$ and the angle of polarization $\varphi$.

To calibrate the mirror used in our catadioptric sensor, the polarization state of the reflected light has to be measured. A rotating polarizer placed between the camera and the mirror is used. The complete sensor (mirror and camera) and the polarizer are placed into a diffuse light environment composed of a backlit cylindrical diffuser (Figure 3). The light intensity of each pixel is linked to the angle of the polarizer and to the polarization parameters by the following equation:

$$
I_{p}(\alpha)=\frac{I}{2}(\rho \cos (2 \alpha-2 \varphi)+1),
$$

where $\alpha$ is the polarizer angle. The purpose of polarization imaging is to compute the three 


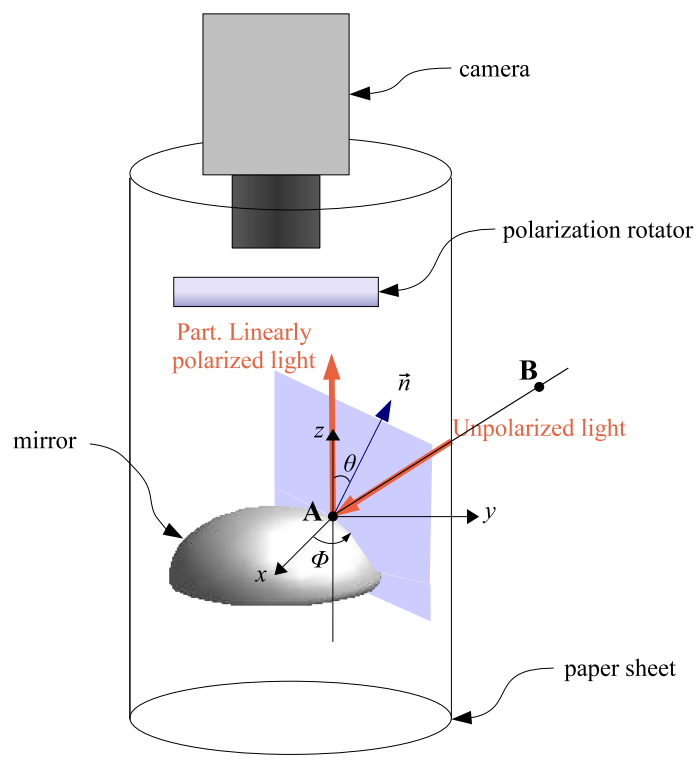

Figure 3: Polarization imaging: after being reflected by the mirror, the light becomes partially linearly polarized.

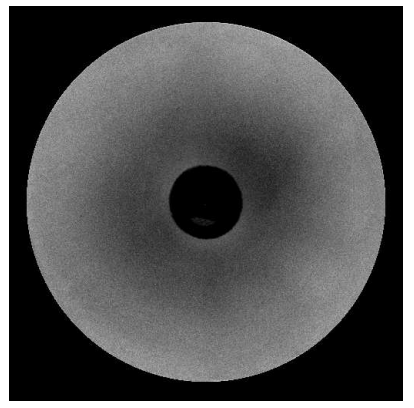

(a)

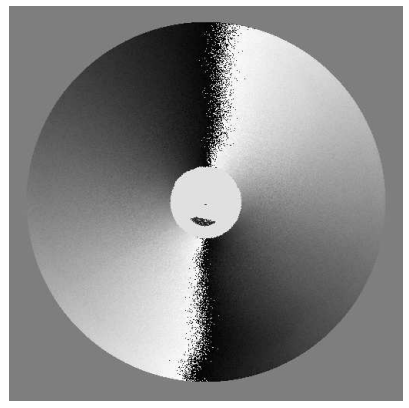

(b)

Figure 4: Images of the polarization parameters that are needed to reconstruct the mirror shape: (a) degree of polarization $(\rho \in[0,1])$, (b) angle of polarization $(\varphi \in[0, \pi])$.

parameters, $I, \varphi$, and $\rho$, by interpolating this formula. Because three parameters need to be determined, at least three images are required. Images are taken with different orientations of the polarizer. To get an automatic calibration of the catadioptric system, a liquid-crystal polarization rotator is used instead of the polarizer. It acts as a rotating polarizer, which has the ability to be electrically controlled. Figure 4 shows an image of the degree and the angle of polarization of a spherical mirror. 


\section{A. The relationship between the polarization parameters and the normals}

Wolff and Boult have demonstrated how to determine constraints on surface normals by using the Fresnel reflectance model [27]. The surface of the mirror is assumed to be continuous and described by a Cartesian expression: $z=f(x, y)$. Therefore, each surface normal is given by the following non-normalized expression:

$$
\vec{n}=\left[\begin{array}{c}
-\frac{\partial f(x, y)}{\partial x} \\
-\frac{\partial f(x, y)}{\partial y} \\
1
\end{array}\right]=\left[\begin{array}{c}
p=\tan \theta \cos \phi \\
q=\tan \theta \sin \phi \\
1
\end{array}\right] .
$$

The aim of the "Shape from polarization" technique is to compute the normals from the angles $\theta$ and $\phi$. By combining the Fresnel formulae and the Snell-Descartes law one can find a relationship between the degree of polarization $\rho$ and the zenith angle $\theta$ [25]. For specular metallic surfaces, the following formula can be applied [28]:

$$
\rho(\theta)=\frac{2 n \tan \theta \sin \theta}{\tan ^{2} \theta \sin ^{2} \theta+|\hat{n}|^{2}}
$$

where $\hat{n}=n(1+i \kappa)$ is the complex refractive index of the mirror. In the case of a perfect specular mirror, the formula (3) can be directly used if the complex refractive index of the material is known. However, if the refractive index is unknown or if the mirror is not perfectly specular a pseudo-refractive index [29] can be applied. This consists of estimating a pseudo-refractive index that best fits the relation between the angle $\theta$ and the degree of polarization $\rho$ measured on a known shape of the same material. Finally, this pseudorefractive index, which has no physical meaning, enables the degree of polarization image of the new object to be computed using the same relation (3).

The azimuth angle $\phi$ is linked to the angle of polarization $\varphi$ since the reflected light becomes partially linearly polarized according to the normal of the plane of incidence. Because our imaging system uses a telecentric lens, orthographic projection onto the sensor is assumed and the azimuth angle $\phi$ can be inferred from the angle of polarization $\varphi$ according to the following equation:

$$
\phi=\varphi \pm \frac{\pi}{2} .
$$




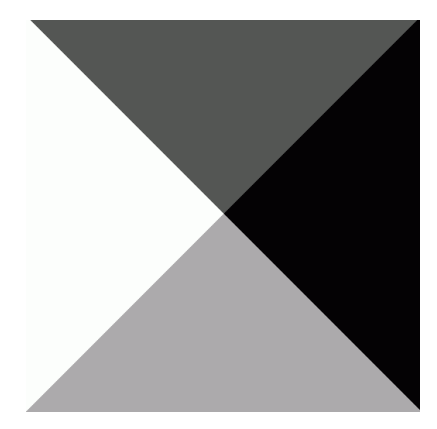

(a)

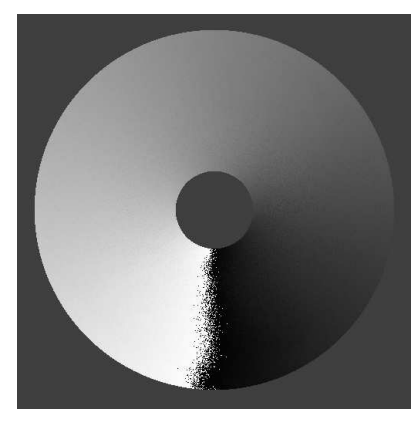

(b)

Figure 5: Disambiguation of the azimuth angle: (a) segmented image ( $\left.I_{\text {quad }} \in\{0,1,2,3\}\right)$, (b) image of the resulting azimuth angle $\phi(\phi \in[-\pi / 2,3 \pi / 2])$.

\section{B. Disambiguation of the normals}

From equations (3) and (4), the surface normals are determined with an ambiguity. Since mirrors used in catadioptric vision are of convex and revolution shape, a segmented image $I_{\text {quad }}$ can be directly computed from the near center of the mirror (Figure 5(a)). This segmented image is an image with four gray levels that represent the four quadrants oriented with an angle in $] 0, \pi / 2[$. The algorithm of the disambiguation process described in [29] is applied with the segmented image $I_{\text {quad }}$ and with the angle of polarization image $\varphi$. The azimut angle $\phi$ is computed as follows:

1. $\phi=\varphi-\frac{\pi}{2}$,

2. $\phi=\phi+\pi$ if $\left[\left(I_{\text {quad }}=0\right) \wedge(\phi \leq 0)\right] \vee\left[I_{\text {quad }}=1\right] \vee\left[\left(I_{\text {quad }}=3\right) \wedge(\phi \geq 0)\right]$,

where $\wedge$ and $\vee$ represent, respectively, the logical operators AND and OR. The result of the disambiguation is presented in Figure $5(\mathrm{~b})$.

\section{Calibration}

To calibrate our imaging system, we use the generic calibration concept introduced by Sturm and Ramalingam [30]. The concept considers an image as a collection of pixels, and each pixel measures the light along a particular 3D ray. Thus, calibration can be seen as the determination of all projection rays and their correspondence with pixels. A $3 \mathrm{D}$ ray is represented here by a couple of points $(\mathbf{A}, \mathbf{B})$ that belong to the ray. The points' 


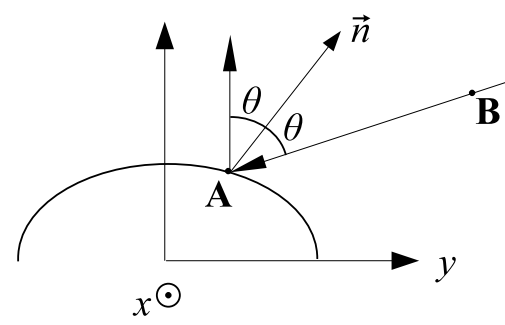

Figure 6: Description of the 3D-ray given by the points $\mathbf{A}$ and $\mathbf{B}$.

coordinates are defined as described in equation (5):

$$
\mathbf{A}=\left[\begin{array}{c}
x_{a} \\
y_{a} \\
z_{a}
\end{array}\right], \mathbf{B}=\left[\begin{array}{l}
x_{b} \\
y_{b} \\
z_{b}
\end{array}\right] .
$$

To calibrate the sensor, let us take point $\mathbf{A}$, that belongs to both the mirror surface and the 3D-ray, to be the first point of the ray (Figure 6). Since orthographic projection is assumed, the $x_{A}$ and $y_{A}$ coordinates can be directly deduced from the $(u, v)$ coordinates of each pixel (up to a scale factor given by the lens). Finally, the computation of the $z_{a}$ coordinate requires the determination of the 3D mirror surface. The 3D surface of the mirror can be computed from the normals (given by polarization imaging) thanks to the FrankotChellappa algorithm [31]. Denoting the Fourier transforms of, respectively, the surface height and the $x, y$ gradients as $\tilde{f}, \tilde{p}$ and $\tilde{q}$, we have:

$$
\forall(u, v) \neq(0,0), \tilde{f}(u, v)=\frac{-j u \tilde{p}-j v \tilde{q}}{u^{2}+v^{2}} .
$$

The three-dimensional surface is obtained by taking the inverse Fourier transform of the former equation. This integration process gives us the surface height of the mirror with a constant of integration. Nevertheless, this constant is not required because of the orthographic projection assumption.

As shown in Figure 6, the second point $\mathbf{B}$ of the ray can be written as:

$$
\mathbf{B}=\mathbf{A}+k\left[\begin{array}{c}
\tan 2 \theta \cos \phi \\
\tan 2 \theta \sin \phi \\
1
\end{array}\right]
$$

where $k$ is a non-null constant. 


\section{EXPERIMENTS}

In the previous section, we showed that the three-dimensional parameters of the mirror, $\theta, \phi$, and $z$, are required to calibrate the catadioptric system, according to the generic calibration concept introduced by Sturm. The azimuth and zenith angles, $\phi$ and $\theta$, are directly given by the measurement of the polarization parameters $(\rho, \varphi)$ of the light reflected by the mirror. The surface height $z$, that represents the 3D shape of the mirror, is obtained by an integration process.

In this section, simulations are first presented to illustrate the influence of the parameters on reconstruction quality. Then, preliminary results on a calibrated spherical mirror show the good accuracy of the three-dimensional parameters measurement by polarization imaging. Experiments on the reconstruction of a real scene are also presented.

\section{A. Simulations}

To simulate the three-dimensional reconstruction error, the synthetic scene introduced in Section 2, Figure 1 is reconstructed thanks to the generic calibration concept. The normals angles $\theta$ and $\phi$ were computed from a perfect parabolic mirror with a $7^{\circ}$ misalignment between the optical axis and the symmetry axis of the mirror. The parameters are then disturbed by adding various levels of Gaussian noise. At each level of noise, the experiment is regenerated 50 times, and the average error reconstruction is computed. Figure 7(a) and Figure 7(b) show, respectively, the reconstruction error of the scene induced by noisy measurement of $\theta$ and $\phi$. The synthetic scene is reconstructed with or without mirror reconstruction, meaning that the integration process from equation (6) is carried out or not (Figure 8).

Figure 7 shows that scene reconstruction is quite sensitive to the measurement of the parameters $\theta$ and $\phi$; on the other hand, the integration process is not required, and we can assume that the $z$ parameter is negligible. In addition, Figure 9 shows the reconstruction error of the scene by only adding Gaussian noise to the mirror height $z$. In this case, the normals are assumed to be perfectly measured and noise is only added on the surface height of the mirror. The reconstruction quality remains good even if the mirror height is very noisy (the mirror height is $1 \mathrm{~cm}$ and the radius is $2 \mathrm{~cm}$ ) or badly reconstructed. 

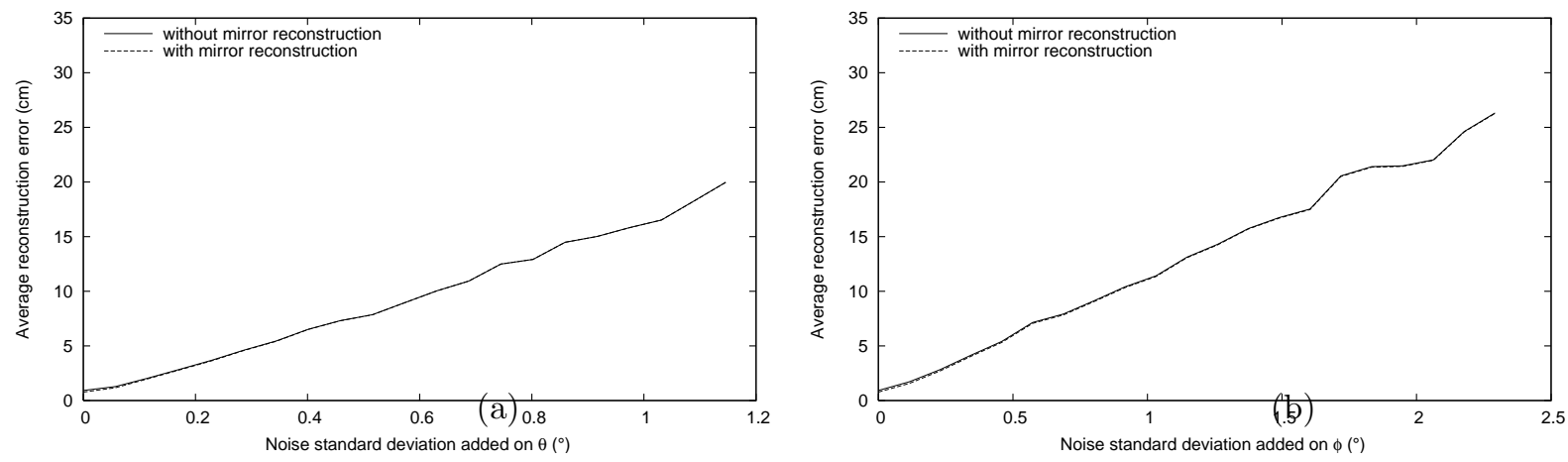

Figure 7: Reconstruction error induced by noisy measurement of the normals parameters: (a) $\theta$ angle, (b) $\phi$ angle.

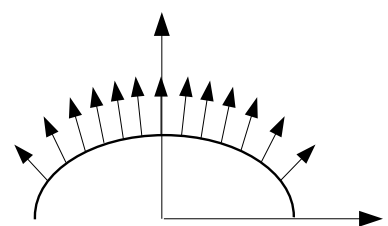

(a)

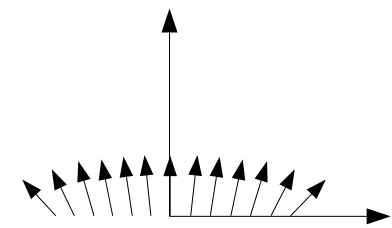

(b)

Figure 8: Three-dimensional parameters used: (a) normals $\theta, \phi$ and surface height $z$, (b) only normals $\theta, \phi$ (without integration process).

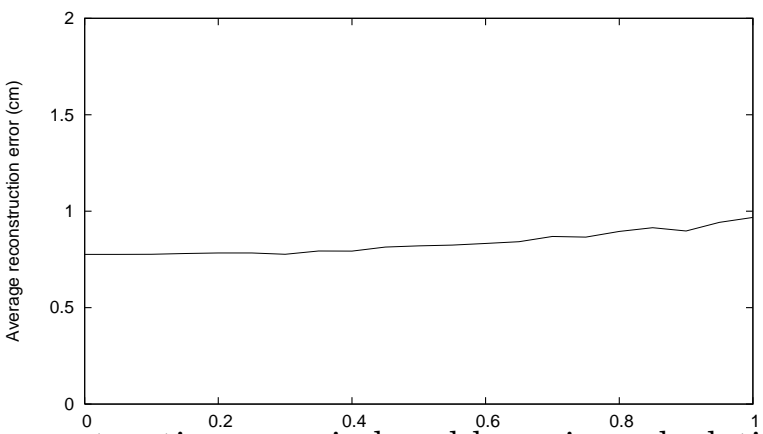

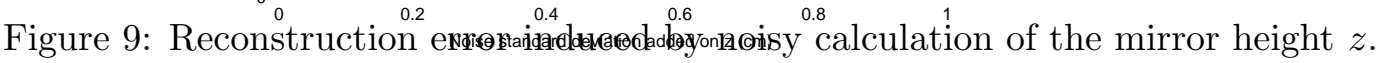




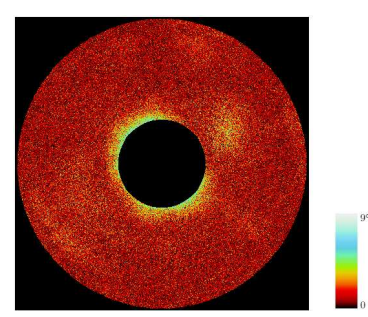

(a)

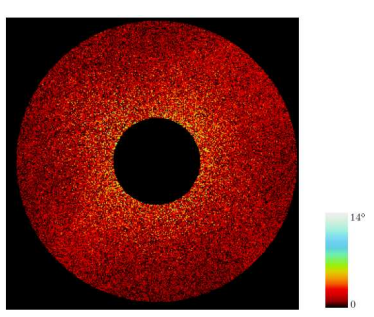

(b)

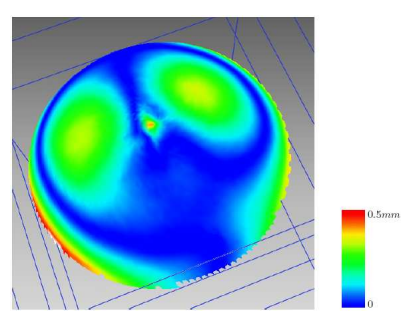

(c)

Figure 10: Measurement errors of the three-dimensional parameters: (a) angle $\theta$, (b) angle $\phi$ and (c) deviation map of the mirror $z$.

\section{B. Preliminary results}

\section{Simulated scene}

Preliminary results were carried out with a catadioptric sensor made of a camera with a telecentric lens and a calibrated spherical mirror $($ radius $=1 \mathrm{~cm})$. Let us notice that our system does not satisfy the single view point constraint. Nevertheless, this property is not required here for the three-dimensional reconstruction of the scene. As described in section 3, our catadioptric sensor is calibrated by measuring the three-dimensional parameters of the mirror with a liquid crystal polarization rotator placed between the camera and the mirror. To evaluate the accuracy of our system, we compare the parameters $(\theta, \phi$, and $z)$ obtained with our system to the theoretical parameters of the mirror (Figure 10).

The mean quadratic errors of the angles $\theta$ and $\phi$ are, respectively, $0.49^{\circ}$ and $1.02^{\circ}$. Figure 11 shows the reconstruction of the synthetic scene by using the calibration made by polarization imaging. Since the mirror is spherical, three-dimensional reconstruction errors increase highly. Nevertheless, the synthetic scene is well reconstructed with an average error of $9.68 \mathrm{~cm}$.

\section{Real scene}

As presented in Figure 12, the three-dimensional reconstruction of a real scene was also carried out. The imaging system is made of a CCD camera with a telecentric lens and a spherical mirror. In order to precisely control its displacement, it has been placed on a precision three axes stage. The $x \times y \times z$ operating area is $1.5 m \times 1.6 m \times 0.4 m$, and the 


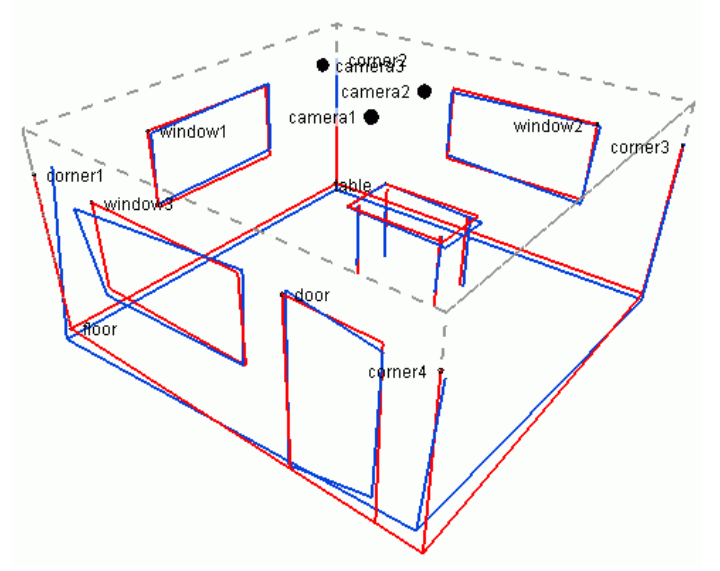

Figure 11: Simulation of the three-dimensional reconstruction by using the calibration done from the polarization imaging.

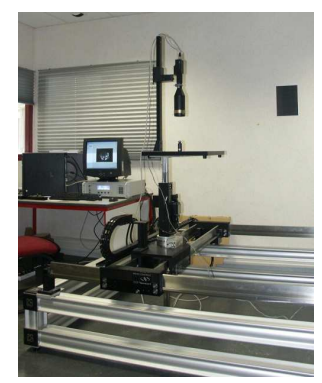

(a)

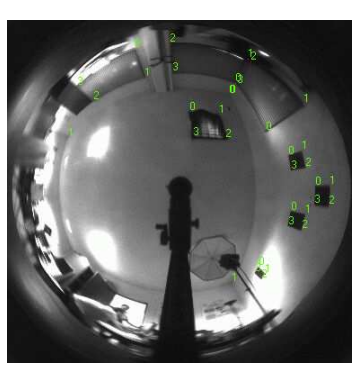

(b)

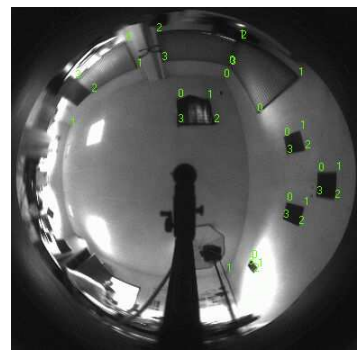

(c)

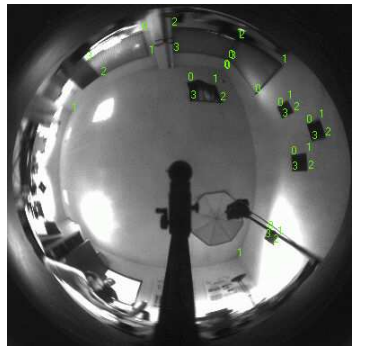

(d)

Figure 12: Three-dimensional reconstruction of a real scene with the catadioptric system: (a) Experimental-set-up, (b,c,d) points of interest picking.

$x \times y \times z$ room size to reconstruct is about $6 m \times 5 m \times 2.5 m$. After calibrating our catadioptric sensor by polarization imaging, 3D points of interest have been triangulated by moving the system.

Once the imaging system is automatically calibrated by polarization imaging, three images with three different known positions have been acquired. Several points of interest were picked on each image: four rectangular targets, two edges of the ceiling, three windows and one fluorescent light (Figure. 12(b,c,d)). The result of the triangulation process presented in Figure 13 shows that the reconstruction is qualitatively satisfactory. The global topology of the scene and the relative distances are respected; right angles appear as (near-) right angles. In fact, the overall reconstruction is as expected, considering: 


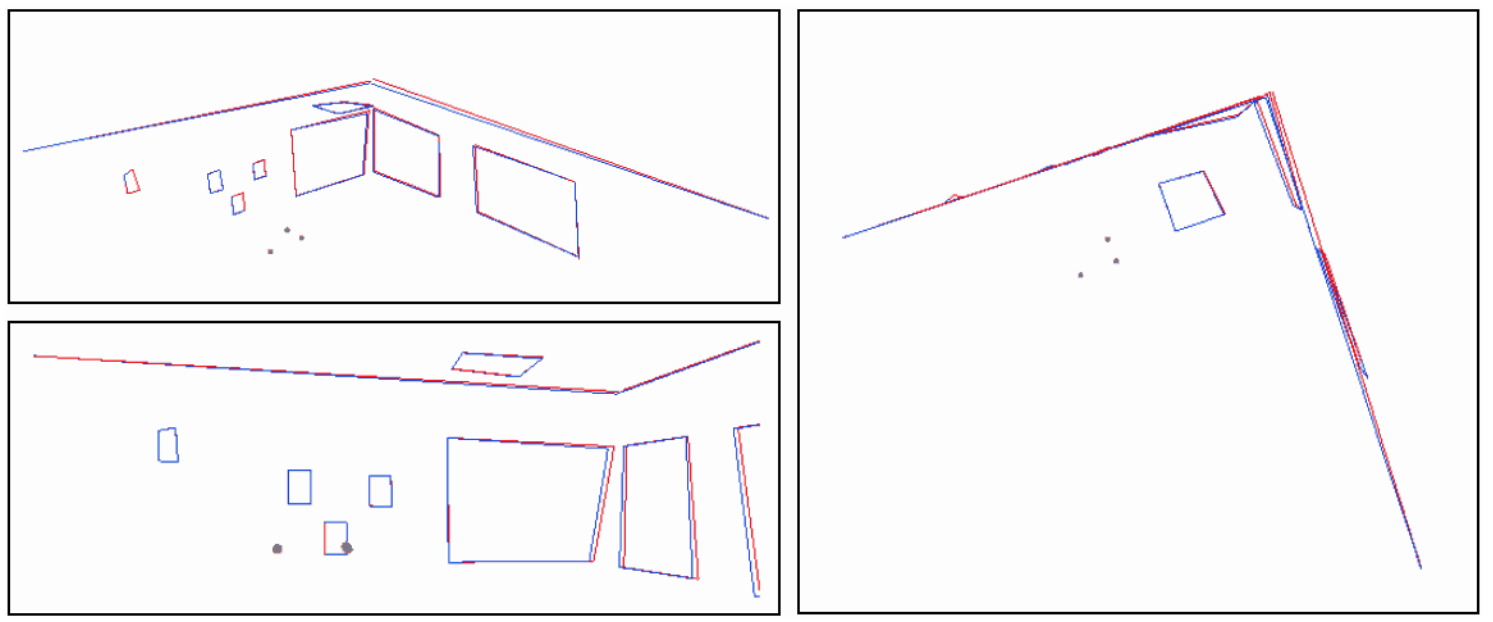

Figure 13: Results of the three-dimensional reconstruction: reconstruction according to the lineareigen and mid-point methods are respectively drawn in red and blue, the gray points represent the 3 positions of the sensor.

- the small displacement of the sensor in comparison with the size of the scene;

- the fact that we only performed linear triangulation without refinement; and

- the reconstruction also depends on the accuracy of point picking (the correspondence has been established manually and a slight error in the location of $2 \mathrm{D}$ points lead to erroneous 3D reconstruction).

\section{CONCLUSION}

In this paper, a new efficient calibration method for catadioptric sensors has been presented. This method is based on the measurement of three-dimensional parameters of the mirror thanks to polarization imaging. The calibration can be performed "in one click" even by a non-specialist, because it only requires an optical apparatus, no image processing and no calibration pattern. Contrary to traditional methods, it deals with misalignment of the sensor and works for any shape mirror (regular or not). Experimental results prove that the sensor is properly calibrated and a satisfactory three-dimensional reconstruction of the scene can be obtained. We have also shown that the 3D-shape of the mirror can be neglected in comparison with the normals orientations. The plateform3D department is currently manufacturing a parabolic mirror and future work will consist of creating a paracatadioptric 
sensor in order to compare our method to other methods known in the literature for real scenes.

[1] S. Baker and S. K. Nayar, "A theory of catadioptric image formation," in International Conference on Computer Vision, pp. 35-42, IEEE Computer Society, (Washington, DC, USA), 1998.

[2] S. Ramalingam, S. K. Lodha, and P. Sturm, "A generic structure-from-motion framework," Computer Vision and Image Understanding 103, pp. 218-228, September 2006.

[3] P. Sturm, "Multi-view geometry for general camera models," in IEEE Computer Vision and Pattern Recognition, 1, pp. 206-212, IEEE Computer Society, (Washington, DC, USA), 2005.

[4] S. Kang, "Catadioptric self-calibration," in IEEE Computer Vision and Pattern Recognition, pp. 1201-1207, 2000.

[5] Y. Yagi, S. Kawato, and S. Tsuji, "Real-time omnidirectional image sensor (copis) for visionguided navigation," IEEE Trans. Robotics and Automation 10, pp. 11-22, February 1994.

[6] J. Fabrizio, J.-P. Tarel, and R. Benosman, "Calibration of panoramic catadioptric sensors made easier," omnivis 00, p. 45, 2002.

[7] C. Geyer and K. Daniilidis, "Structure and motion from uncalibrated catadioptric views," IEEE Computer Vision and Pattern Recognition 01, pp. 279-293, 2001.

[8] C. Geyer and K. Daniilidis, "Paracatadioptric camera calibration," IEEE Trans. Pattern Analysis and Machine Intelligence 24(5), pp. 687-695, 2002.

[9] C. Geyer and K. Daniilidis, "Catadioptric camera calibration," International Conference on Computer Vision 01, p. 398, 1999.

[10] J. Barreto and H. Araujo, "Geometric properties of central catadioptric line images," in European Conference on Computer Vision, 2002.

[11] X. Ying and Z. Hu, "Catadioptric camera calibration using geometric invariants," IEEE Trans. Pattern Analysis and Machine Intelligence 26, pp. 1260-1271, October 2004.

[12] M. D. Grossberg and S. K. Nayar, "A general imaging model and a method for finding its parameters," in International Conference on Computer Vision, 2, pp. 108-115, 2001.

[13] D. Aliaga, "Accurate catadioptric calibration for real-time pose estimation of room-size environments," pp. I: 127-134, 2001. 
[14] R. Swaminathan, M. D. Grossberg, and S. K. Nayar, "Caustics of catadioptric cameras," iccv 02, p. 2, 2001.

[15] C. Mei and P. Rives, "Single view point omnidirectional camera calibration from planar grids," in IEEE International Conference on Robotics and Automation, April 2007.

[16] S. Ramalingam, P. Sturm, and S. Lodha, "Theory and calibration algorithms for axial cameras," in Proceedings of the Asian Conference on Computer Vision, Hyderabad, India, I, pp. 704-713, jan 2006.

[17] J. P. Barreto and H. Araujo, "Paracatadioptric camera calibration using lines," International Conference on Computer Vision 02, pp. 1359-1365, 2003.

[18] B. Vanderportaele, M. Cattoen, P. Marthon, and P. Gurdjos, "A new linear calibration method for paracatadioptric cameras," International Conference on Pattern Recognition 4, pp. 647$651,2006$.

[19] R. Hartley and P. Sturm, "Triangulation," Computer Vision and Image Understanding 68(2), pp. 146-157, 1997.

[20] J.-J. Gonzalez-Barbosa, Vision panoramique pour la robotique mobile : stéréovision et localisation par indexation d'images. PhD thesis, Université Toulouse III, 2004.

[21] J.-J. Gonzalez-Barbosa and S. Lacroix, "Fast dense panoramic stereovision," pp. 1210-1215, 18-22 April 2005.

[22] S. Ieng and R. Benosman, "Geometric construction of the caustic curves for catadioptric sensors," pp. V: 3387-3390, 2004.

[23] R. Pless, "Using many cameras as one," in IEEE Computer Vision and Pattern Recognition, 2, pp. 587-593, 2003.

[24] L. B. Wolff, "Polarization-based material classification from specular reflection," IEEE Trans. Pattern Analysis and Machine Intelligence 12, pp. 1059-1071, November 1990.

[25] D. Miyazaki, M. Kagesawa, and K. Ikeuchi, "Transparent surface modeling from a pair of polarization images," IEEE Trans. Pattern Analysis and Machine Intelligence 26, pp. 73-82, January 2004.

[26] S. Rahmann, "Reconstruction of quadrics from two polarization views," in Iberian Conference on Pattern Recognition and Image Analysis (IbPRIA03), Springer, LNCS 2652, Mallorca, Spain, pp. 810-820, June 2003.

[27] L. B. Wolff and T. E. Boult, "Constraining object features using a polarization reflectance 
model," IEEE Trans. Pattern Analysis and Machine Intelligence 13, pp. 635-657, July 1991.

[28] O. Morel, C. Stolz, F. Meriaudeau, and P. Gorria, "Three-dimensional inspection of highlyreflective metallic objects by polarization imaging," Electronic Imaging Newsletter 15(2), p. 4, 2005.

[29] O. Morel, C. Stolz, F. Meriaudeau, and P. Gorria, "Active lighting applied to 3d reconstruction of specular metallic surfaces by polarization imaging," Applied Optics 45, pp. 4062-4068, June 2006.

[30] P. Sturm and S. Ramalingam, "A generic concept for camera calibration," in European Conference on Computer Vision, 2, pp. 1-13, Springer, (Prague, Czech Republic), May 2004.

[31] R. Frankot and R. Chellappa, "A method for enforcing integrability in shape from shading algorithms," IEEE Trans. Pattern Analysis and Machine Intelligence 10, pp. 439-451, July 1988.

[32] Plateform3D Department - 3 Dimensional Solid Modelling and Prototyping http://www.plateform3d.com 